\title{
First report of Vialaea minutella in Australia, its association with mango branch dieback and systematic placement of Vialaea in the Xylariales
}

\author{
A. R. McTaggart • K. R. Grice • R. G. Shivas
}

Received: 4 February 2013 /Accepted: 5 April 2013 /Published online: 8 May 2013

(C) Australasian Plant Pathology Society Inc. 2013

\begin{abstract}
Vialaea minutella was consistently isolated from infected mango trees showing branch dieback symptoms in northern Queensland. The fungus was identified by morphology and confirmed with molecular sequence data. This is the first report of $V$. minutella in Australia. The systematic position of Vialaea was confirmed to be in the Xylariales based on reconstructed LSU sequence data.
\end{abstract}

Keywords Branch dieback · Mangifera indica .

Sordariomycetes

In 2012, branch dieback was observed on mango (Mangifera indica cv. Kensington Pride) trees at a property near Mareeba, Queensland. Isolations from branches showing dieback symptoms (Fig. 1a) on two separate occasions yielded slowgrowing colonies between 2 and $5 \mathrm{~cm}$ diam. on potatodextrose agar after 8 weeks incubated under $12 \mathrm{~h}$ near ultraviolet light / $12 \mathrm{~h}$ dark at $25^{\circ} \mathrm{C}$. The colonies were greenish black, adpressed with little aerial mycelium and irregular dendritic or coralloid margins (Fig. 1b). A few perithecia that contained asci with isthmoid ascospores (Fig. 1c-e) were

\footnotetext{
A. R. McTaggart $(\bowtie)$

Department of Agriculture, Fisheries and Forestry, Plant Pathology Herbarium, Agri-Science Queensland, GPO Box 267, Brisbane, Queensland 4001, Australia

e-mail: alistair.mctaggart@gmail.com

K. R. Grice

Department of Agriculture, Fisheries and Forestry, Agri-Science Queensland, PO Box 1054, Mareeba, Queensland 4880, Australia

R. G. Shivas

Department of Agriculture, Fisheries and Forestry, Plant Pathology Herbarium, Biosecurity Queensland, GPO Box 267, Brisbane, Queensland 4001, Australia
}

scattered and embedded within the colonies. Asci were 8 spored, 130-200 $\times 12-15 \mu \mathrm{m}$ with bifusoid, mostly biseptate ascospores that measured $80-100 \times 4-5 \mu \mathrm{m}$, usually $2-3 \mu \mathrm{m}$ in the middle of the spore. The isolates were identified as Vialaea minutella, which was first described by Petrak (1952) from a Hawaiian sample on mango. The description of the lectotype provided by Cannon (1995) had similar spore sizes, the asci were $154-224 \times 10.5-12.5$ and the ascospores were 96-112 $\times 4-4.5 \mu \mathrm{m}$. The living cultures were deposited in the DAFF Plant Pathology Herbarium as BRIP 56959 and BRIP 56960.

Vialaea Sacc., a pathogen of wood with distinctive isthmoid or bifusiform ascospores, had an ambiguous systematic placement within the Sordariomycetes. It is known from two species, the type $V$. insculpta on Ilex and $V$. minutella on mango. Cannon (1995) placed Vialaea in the family Vialaceaceae, which he tentatively included in the Diaporthales based on structure of the stromata and ascomata as well as its nutritional strategy as a weak parasite. Redlin (1989) considered Vialaea to belong to the Amphisphaeriaceae in the Xylariales, based on the presence of an amyloid annulus in the ascus. Shoemaker et al. (2013) included Vialaea in the Xylariales based on a systematic analysis of the Small Subunit region and morphology of the ascal annulus.

DNA was extracted from two cultured isolates using the Qiagen Puregene Kit. The Internal Transcribed Spacer (ITS) region of rDNA was amplified with ITS1/ITS4 (White et al. 1990) and the Large Subunit (LSU) region was amplified with LROR/LR6 (Vilgalys and Hester 1990). Reactions were performed with Phusion mastermix (Finnzymes) according to the manufacturer's protocol and with an annealing temperature of $60^{\circ} \mathrm{C}$. Clean-up and direct sequencing of the PCR product was completed by Macrogen (Korea). Sequences from the two isolates differed by two base pairs in the ITS region and were 
Fig. 1 Vialaea minutella (BRIP56960). a Branch dieback symptoms, b cultural characteristics, $\mathbf{c}-\mathbf{d}$ asci, $\mathbf{e}$ isthmoid shaped ascospore. Scale $=10 \mu \mathrm{m}$
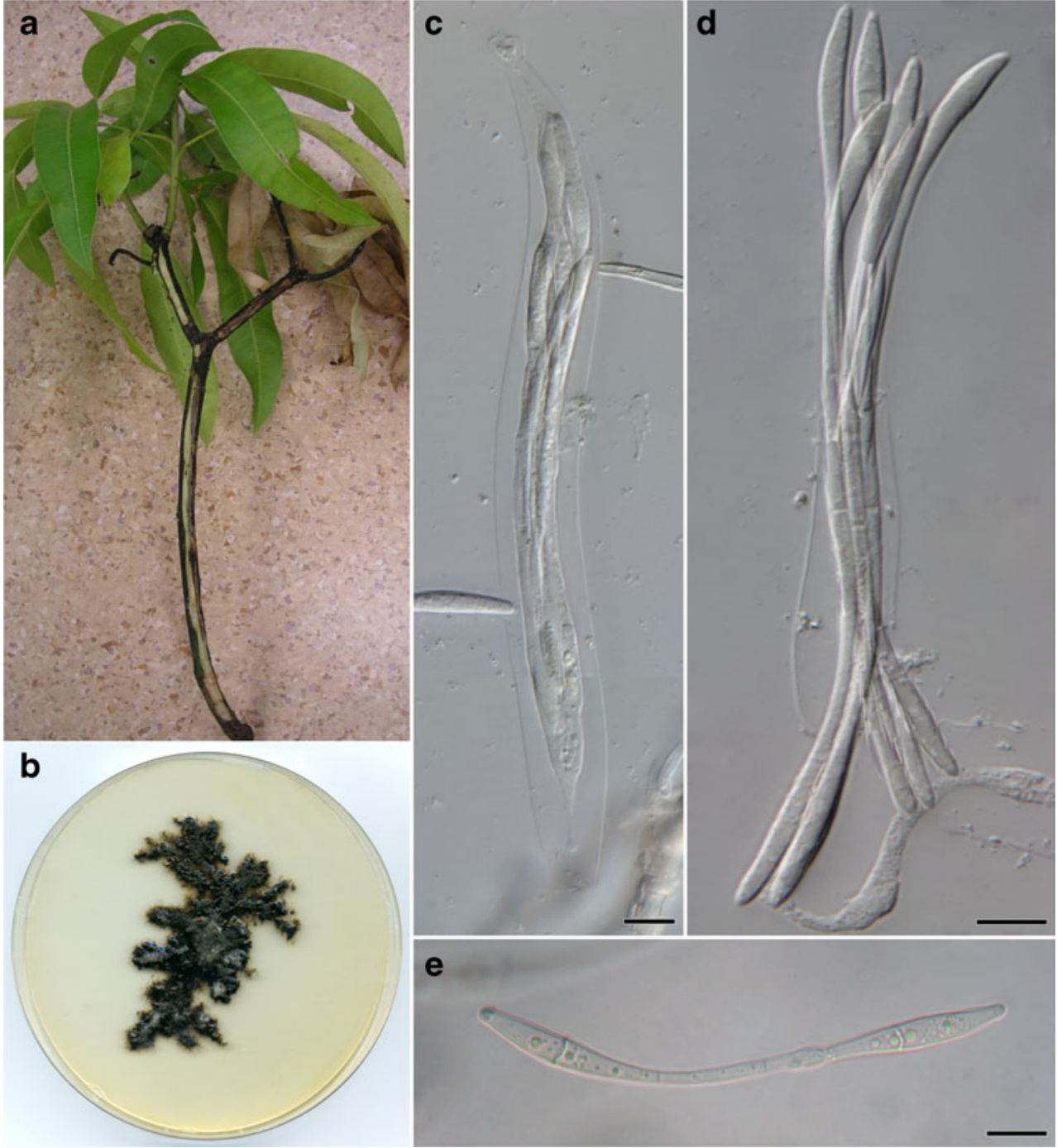

identical in the LSU region. Sequences have been deposited in GenBank (BRIP 56959 ITS: KC181924, LSU: KC181926; BRIP 56960 ITS: KC181925, LSU: KC181927). A BLAST search on the ITS and LSU regions returned a $95 \%$ and $98 \%$ match to Vialaea insculpta (GenBank JX139726) respectively.

A maximum likelihood search of the LSU region was done in RAxML (Stamatakis 2006) and PhyML 3.0 (Guindon et al. 2010). Taxa were selected from the Sordariomycetes dataset provided in the study of Schoch et al. (2009). A taxon each from the Dothideomycetes and Laboulbeniomycetes were used as outgroups. GTRGAMMA was specified as the model of evolution in the two reconstruction programs. The RAxML analyses were run with a rapid Bootstrap analysis (command $-\mathrm{f}$ a) using a random starting tree and 1,000 maximum likelihood bootstrap replicates. The PhyML analyses were implemented using the ATGC bioinformatics platform (available at: http://www.atgcmontpellier.fr/phyml/), with SPR tree improvement, and support obtained from an approximate likelihood ratio test (Anisimova et al. 2011). Identical topologies were recovered in the two maximum likelihood analyses (Fig. 2). The Xylariales formed a well supported group (99\% aRLT), as did the sub-classes, Sordariomycetidae and Hypocreomycetidae, which were recovered in a systematic analysis on the Sordariomycetes by Zhang et al. (2006). The three isolates of Vialaea were monophyletic within the Xylariales and were sister to other known families, Amphisphaeriaceae and Xylariaceae. 


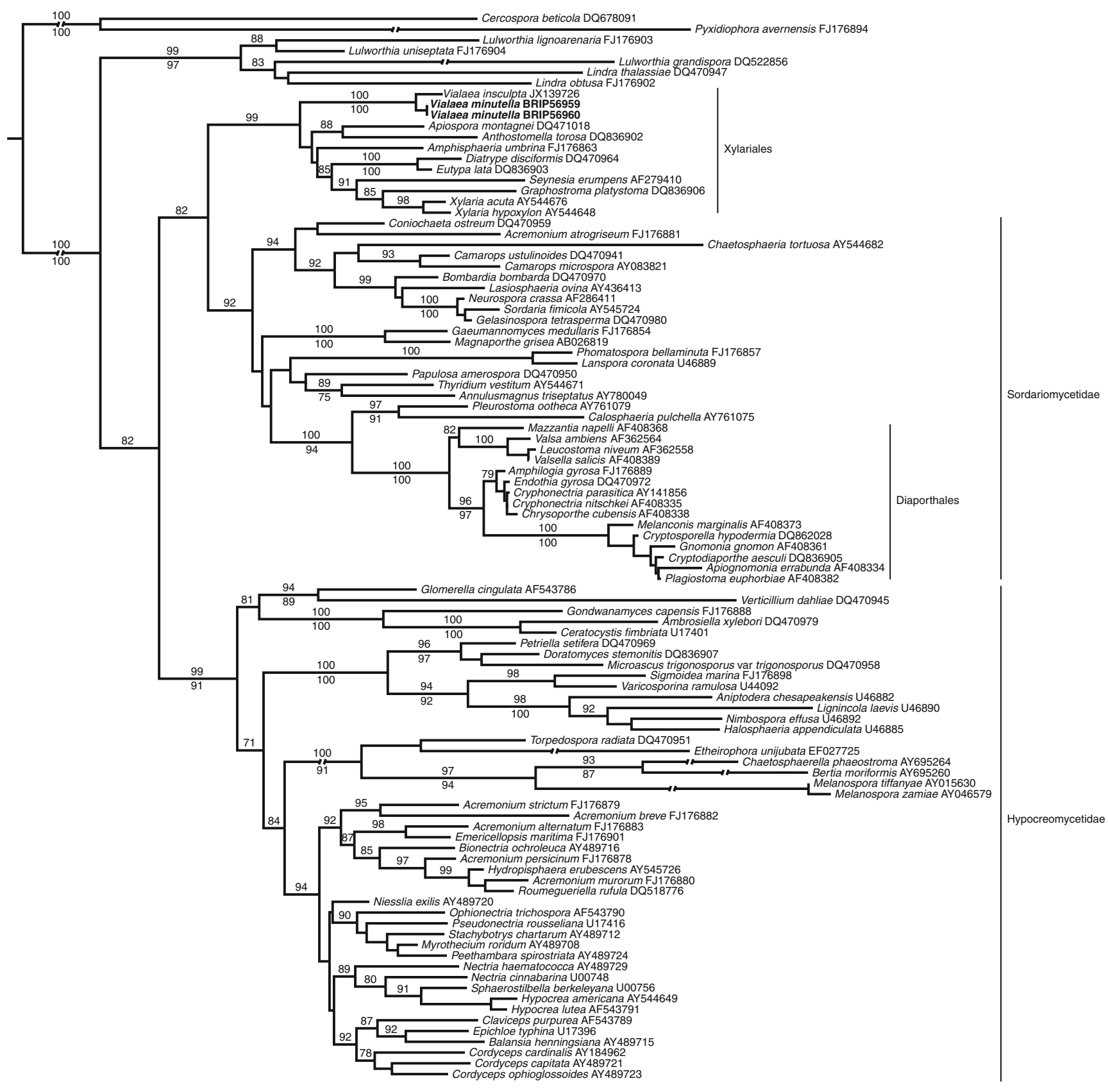

0.2

Fig. 2 Phylogram recovered from a maximum likelihood search in PhyML with an alignment of the LSU region. aRLT values $>70 \%$

This is the first report of Vialaea minutella in Australia. The higher classification of Vialaea in the Xylariales, rather than the Diaporthales, as recovered by Shoemaker et al. shown above nodes and bootstrap values $>70 \%$ from a maximum likelihood search in RAxML shown below nodes

(2013), is supported by LSU data in this study. A separate family, the Vialaceaceae as proposed by Cannon (1995), is also supported. 


\section{References}

Anisimova M, Gil M, Dufayard JF, Dessimoz C, Gascuel O (2011) Survey of branch support methods demonstrates accuracy, power, and robustness of fast likelihood-based approximation schemes. Syst Biol 60:685-699

Cannon PF (1995) Studies on fungi with isthmoid ascospores: the genus Vialaea, with the description of the new family Vialaeaceae. Mycol Res 99:367-373

Guindon S, Dufayard JF, Lefort V et al (2010) New algorithms and methods to estimate maximum-likelihood phylogenies: assessing the performance of PhyML 3.0. Syst Biol 59:307-321

Petrak F (1952) Ein Beitrag zur Pilzflora von Hawai. Sydowia 6:363-371

Redlin SC (1989) Observations of Vialaea insculpta (Amphisphaeriaceae). Sydowia 41:296-307

Schoch CL, Sung GH, Lopez-Giraldez F et al (2009) The Ascomycota tree of life: a phylum-wide phylogeny clarifies the origin and evolution of fundamental reproductive and ecological traits. Syst Biol 58:224-239

Shoemaker RA, Hambleton S, Liu M (2013) Vialaea insculpta revisited. N Am Fungi

Stamatakis A (2006) RAxML-VI-HPC: maximum likelihood-based phylogenetic analyses with thousands of taxa and mixed models. Bioinformatics 22:2688-2690

Vilgalys R, Hester M (1990) Rapid genetic identification and mapping of enzymatically amplified ribosomal DNA from several Cryptococcus species. J Bacteriol 172:4238-4246

White TJ, Bruns TD, Lee S, Taylor JW (1990) Amplification and direct sequencing of fungal ribosomal RNA genes for phylogenetics. In: Innis MA, Gelfand DH, Sninsky JJ, White TJ (eds) PCR protocols: a guide to methods and applications. Academic Press, Inc, San Diego, pp 315-322

Zhang N, Castlebury LA, Miller AN et al (2006) An overview of the systematics of the Sordariomycetes based on a four-gene phylogeny. Mycologia 98:1076-1087 